\title{
Theory and Computation for the Microstructure Near the Interface Between Twinned Layers and a Pure Variant of Martensite
}

\author{
Bo Li \\ Mitchell Luskin* \\ Department of Mathematics \\ School of Mathematics \\ University of Maryland \\ University of Minnesota \\ College Park, MD 20742 USA \\ Minneapolis, MN 55455 USA
}

\begin{abstract}
.
We report results on a finite element simulation of a needle-like martensitic microstructure observed in biaxial loading experiments conducted by Chu and James on single crystals of the shape-memory alloy $\mathrm{Cu}-14 \mathrm{at} . \% \mathrm{Al}-3.9 \mathrm{at} . \% \mathrm{Ni}$. This microstructure was observed near an interface between twinned layers of two variants and a pure variant of martensite. We used a geometrically nonlinear theory of martensite to model and compute the complex microstructure by energy minimization with a boundary condition that is compatible with the microstructure. Our computational model has yielded multiple metastable states that depend on the initial state of our energy-minimizing iteration. The simulated twinned layers form branches and bend as they approach the laminate-single variant interface in good agreement with the experiment of Chu and James.
\end{abstract}

Keywords: Martensite; Microstructure; Finite element method

* Corresponding author. Tel.: 1 612 6256565; fax: 1612 6262017; email: luskin@math.umn.edu. 


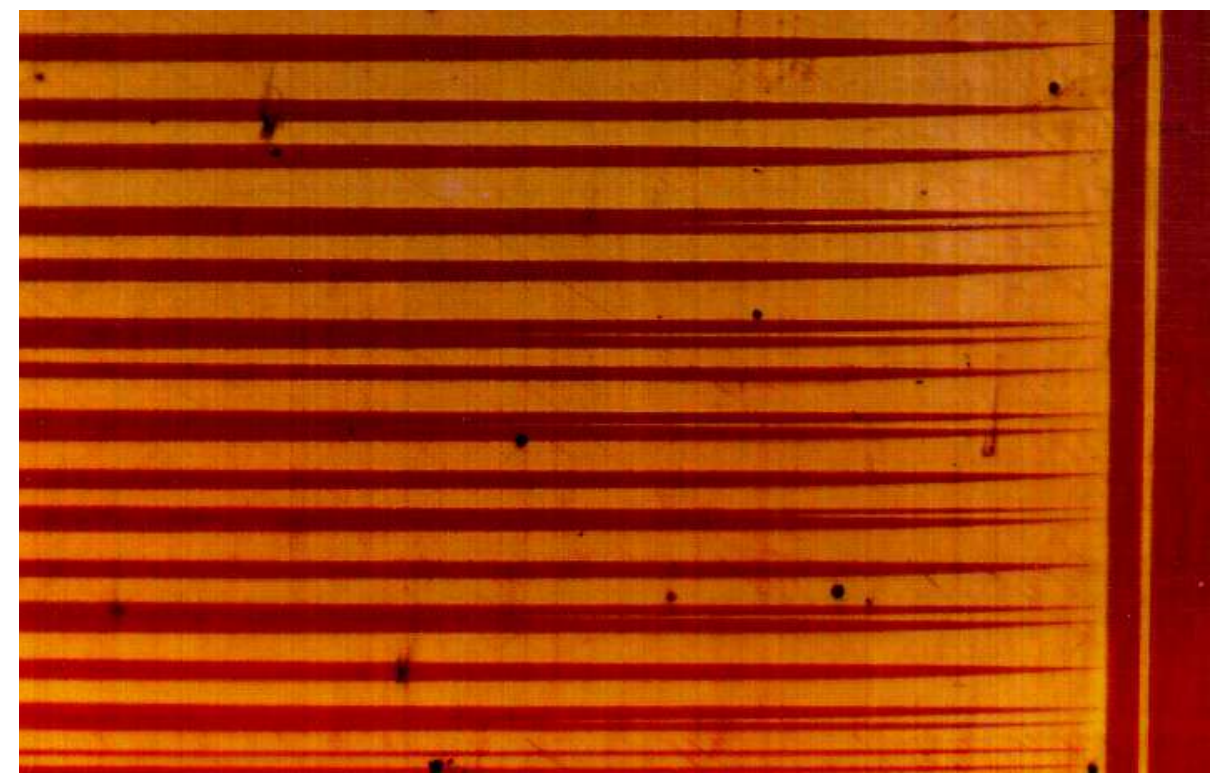

Fig. 1. Photomicrograph of a needle-like microstructure near a laminate-single variant interface (Chu and James). Field of view: $2.5 \mathrm{~mm} \times 1.6 \mathrm{~mm}$.

\section{Introduction.}

A needle-like microstructure near an interface between twinned layers of two variants and a pure variant of martensite was observed by Chu and James in a series of biaxial loading experiments on single crystals of the shape-memory alloy Cu-14at.\%Al-3.9at.\%Ni [1-4] (see Fig. 1). The alloy was cut so that the face of the crystal had normal (100) in the cubic austenite basis. In this article, we report our results of a finite element simulation of the needle-like microstructure aimed at understanding the mechanism and the physical properties of the microstructure.

Our simulation used a continuum model based on a geometically nonlinear theory of martensite [5-9]. Although the Cu-14at.\%Al-3.9at.\%Ni alloy has six orthorhombic variants, only two compound variants were observed in this experiment. Some theoretical validation for this observation has been given in $[1,4,10]$. Consequently, we developed a two-dimensional model for this experiment based on energy minimization with a rotationally invariant doublewell energy density that has linear elastic moduli close to those of the $\mathrm{Cu}-14 \mathrm{at} . \% \mathrm{Al}-3.9 \mathrm{at} . \% \mathrm{Ni}$ alloy [11]. 
We used the finite element method and a variation of the Polak-Ribière conjugate gradient algorithm to solve the minimization problem. Our simulation exhibited the branching and bending of the needles observed in the Chu-James experiment. Other models and numerical algorithms for this phenomena have been reported in $[12,13]$.

\section{The Continuum Model.}

In a fixed orthonormal basis $\left\{e_{1}, e_{2}\right\}$ of $\mathbb{R}^{2}$, the two-dimensional transformation matrices for the two variants participating in the microstructure are

$$
U_{1}=\left(\begin{array}{cc}
\alpha & 0 \\
0 & \beta
\end{array}\right) \quad \text { and } \quad U_{2}=\left(\begin{array}{cc}
\beta & 0 \\
0 & \alpha
\end{array}\right)
$$

where $\alpha=1.0619$ and $\beta=1.0232$ are the transformation strains [2-5]. From the experimental observation, we assume that the laminate in the microstructure is composed of two compatible deformation gradients $Q U_{1}$ and $U_{2}$ for some rotation matrix $Q$ with the volume fractions $\lambda$ and $1-\lambda$. The rotation matrix $Q$ and the unit vector $n$ normal to the parallel layers are determined by the equation for a coherent interface between twinned variants

$$
Q U_{1}-U_{2}=a \otimes n
$$

for some vector $a \in \mathbb{R}^{2}[2-5,9]$. The experimental observation also suggests that we assume that the gradient of the homogeneous deformation on the other side of the interface to be $R_{\lambda} U_{2}$ for some rotation matrix $R$ which depends on the volume fraction $\lambda$. We approximate the interface separating the laminated region from the homogeneous region by a planar interface separating the two homogeneous states $\lambda Q U_{1}+(1-\lambda) U_{2}$ and $R_{\lambda} U_{2}$. The compatibility

condition for this laminate-single variant interface is that the rotation matrix $R_{\lambda}$ and the unit vector $m_{\lambda} \in \mathbb{R}^{2}$ normal to the planar interface must satisfy the equation

$$
R_{\lambda} U_{2}-\left[\lambda Q U_{1}+(1-\lambda) U_{2}\right]=b_{\lambda} \otimes m_{\lambda}
$$

for some vector $b_{\lambda} \in \mathbb{R}^{2}[2-5,9]$. 
For a given volume fraction $\lambda \in(0,1)$, we found the following solution $(Q, a, n)$ of equation (1) and the solution $\left(R_{\lambda}, b_{\lambda}, m_{\lambda}\right)$ of the corresponding equation (2) [14]:

$$
\begin{gathered}
Q=\left(\begin{array}{cc}
\xi & \eta \\
-\eta & \xi
\end{array}\right), \quad a=\sqrt{2} \eta\left(\begin{array}{c}
\beta \\
-\alpha
\end{array}\right), \quad n=\frac{\sqrt{2}}{2}\left(\begin{array}{c}
1 \\
1
\end{array}\right), \\
R_{\lambda}=I+\left(\lambda a \otimes n+b_{\lambda} \otimes m_{\lambda}\right) U_{2}^{-1}, \quad b_{\lambda}=-\frac{\lambda \eta \sqrt{2\left(1+\theta^{2}\right)}}{\xi^{2}+\lambda^{2} \eta^{2}}\left(\begin{array}{c}
\beta(1+(1+\lambda) \eta) \\
\alpha(1-(1+\lambda) \eta)
\end{array}\right), \\
m_{\lambda}=-\frac{1}{\sqrt{2\left(1+\theta^{2}\right)}}\left(\begin{array}{c}
\theta-1 \\
\theta+1
\end{array}\right),
\end{gathered}
$$

where $\xi=2 \alpha \beta /\left(\alpha^{2}+\beta^{2}\right), \eta=\left(\alpha^{2}-\beta^{2}\right) /\left(\alpha^{2}+\beta^{2}\right)$, and $\theta=(1-\lambda) \eta$. We have that $m_{\lambda} \cdot n=-\theta / \sqrt{1+\theta^{2}}$, so the maximum deviation of the normal to the laminate-single variant interface $m_{\lambda}$ from the tangent to the layers of the laminate $n$ is about $2.14^{\circ}$.

We used the geometrically nonlinear theory of thermoelasticity and computed the deformation $y(x)$ of a reference domain $\Omega_{\lambda}$ which minimizes the elastic energy $[5,7,9]$

$$
\mathcal{E}_{\lambda}(y)=\int_{\Omega_{\lambda}} \phi(\nabla y(x), T) d x
$$

where $\phi(F, T)$ is the elastic energy density, $\nabla y(x)$ is the deformation gradient, and $T$ is the temperature. We used the energy density $\phi(F, T)$ given by [11]

$$
\phi(F, T)= \begin{cases}\phi_{0}\left(C_{11}, C_{22}, C_{12}, T\right) & \text { if } C_{11} \geq C_{22}, \\ \phi_{0}\left(C_{22}, C_{11}, C_{12}, T\right) & \text { if } C_{11}<C_{22}\end{cases}
$$

with

$\phi_{0}\left(C_{11}, C_{22}, C_{12}, T\right)=\frac{\sigma_{1}}{2}\left(C_{11}-\alpha^{2}\right)^{2}+\frac{\sigma_{2}}{2}\left(C_{22}-\beta^{2}\right)^{2}+\frac{\sigma_{3}}{2}\left(C_{11}+C_{22}-\alpha^{2}-\beta^{2}\right)^{2}+\frac{\sigma_{4}}{2} C_{12}^{2}$, where $C=\left(C_{i j}\right)=F^{T} F$ is the Cauchy-Green strain tensor of the deformation gradient $F$ and the moduli at room temperature in gigapascals are $\sigma_{1}=27.5, \sigma_{2}=37.2, \sigma_{3}=-11.8$, and $\sigma_{4}=16.7$. We note that the energy density $\phi(F, T)$ is minimized by the transformation matrices $R U_{1}$ and $R U_{2}$ where $R$ is any rotation matrix.

We chose the reference domain $\Omega_{\lambda}$ to be a parallelogram with one vertex at the origin and with sides of length $L_{1}$ and $L_{2}$ perpendicular to $\pm n$ and $\pm m_{\lambda}$, respectively (see Fig. 2). On 


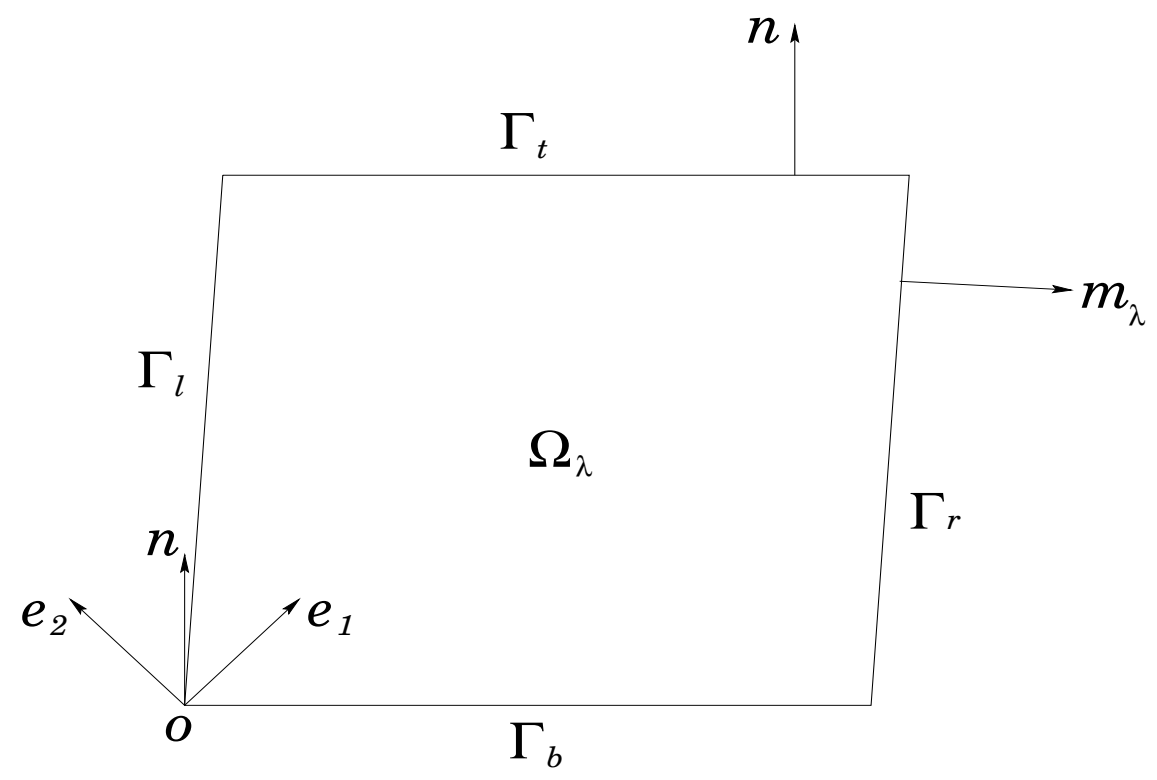

Fig. 2. The reference domain $\Omega_{\lambda}$.

the right side $\Gamma_{r}, y(x)$ was constrained by the compatibility condition for the laminate-single variant interface (2) to satisfy

$$
y(x)=R_{\lambda} U_{2} x+d_{\lambda} \quad \text { for all } x \in \Gamma_{r},
$$

where $d_{\lambda}=\left(d_{\lambda 1}, d_{\lambda 2}\right) \in \mathbb{R}^{2}$ is a constant vector. On the left side $\Gamma_{l}, y(x)$ was constrained to be a piecewise affine deformation that matches continuously a laminate of $N_{\text {layers }}$ periodic layers with gradients taking the values of the transformation matrices $Q U_{1}$ and $U_{2}$ with the volume fractions $\lambda$ and $1-\lambda$, respectively. On the sides $\Gamma_{b}$ and $\Gamma_{t}$, we imposed the periodicity of $\nabla y(x)$ by the constraint

$$
y\left(x+L_{2} m_{\lambda}^{\perp}\right)=y(x)+L_{2} R_{\lambda} U_{2} m_{\lambda}^{\perp}, \quad \text { for all } x \in \Gamma_{b}
$$

where $L_{2}$ is the length of $\Gamma_{l}$ and $m_{\lambda}^{\perp}$ is a unit vector perpendicular to $m_{\lambda}$.

\section{Numerical Methods and Graphics.}

We used the bilinear isoperimetric finite element on a uniform mesh with four-point Gaussian quadrature to discretize the continuum problem [15], and we used a variation of the Polak-Ribière conjugate gradient algorithm to minimize the corresponding multi-variable function. 


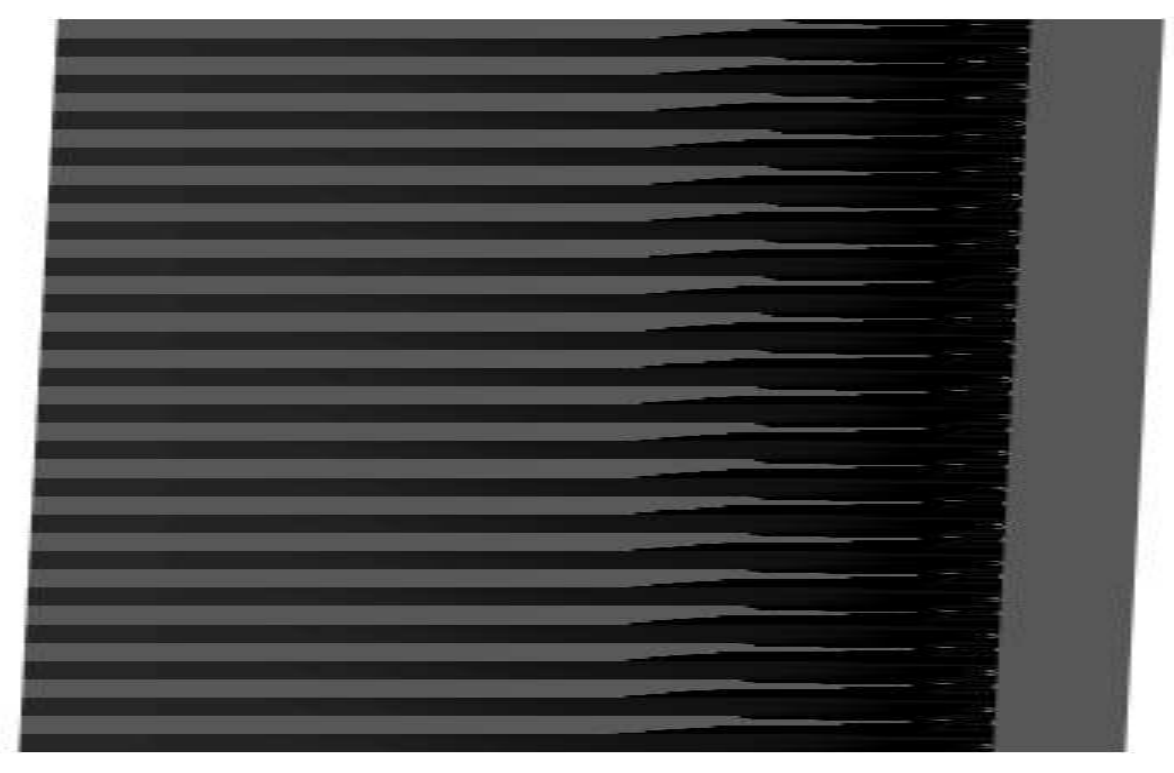

Fig. 3. Bent needles. Parameters: $\lambda=.5, L_{1}=.061, L_{2}=1, N_{\text {layers }}=20, N_{1}=200$, $N_{2}=400, d_{\lambda}=(.00184, .00171)$, and niter $=300$.

To visualize the deformation gradient, we used a gray scale which shaded a region white where the Cauchy-Green stretch tensor $\left(F^{T} F\right)^{1 / 2}$ of the deformation gradient $F$ is close to $U_{1}$ and black where the Cauchy-Green stretch tensor $\left(F^{T} F\right)^{1 / 2}$ of the deformation gradient $F$ is close to $U_{2}$. We note that our computational results are displayed in the reference configuration, whereas the photomicrograph in Fig. 1 exhibits the deformed configuration.

The parameters in our computations were the volume fraction $\lambda \in(0,1)$, the length $L_{1}$ of $\Gamma_{b}$, the length $L_{2}$ of $\Gamma_{l}$, the number of layers $N_{\text {layers }}$ of the laminated deformation in the boundary data for $\Gamma_{l}$, the number of subintervals $N_{1}$ and $N_{2}$ (or equivalently the mesh size $h_{1}=L_{1} / N_{1}$ and $\left.h_{2}=L_{2} / N_{2}\right)$, the vector $d_{\lambda}$ in (3), and the maximal number of iterations niter of the numerical method.

\section{Computational Results.}

Our first computational result is shown in Fig. 3. In this computation, the initial deformation in the conjugate gradient iteration corresponds to the deformation which is identical to the laminated deformation in the boundary data throughout the domain except in a strip 


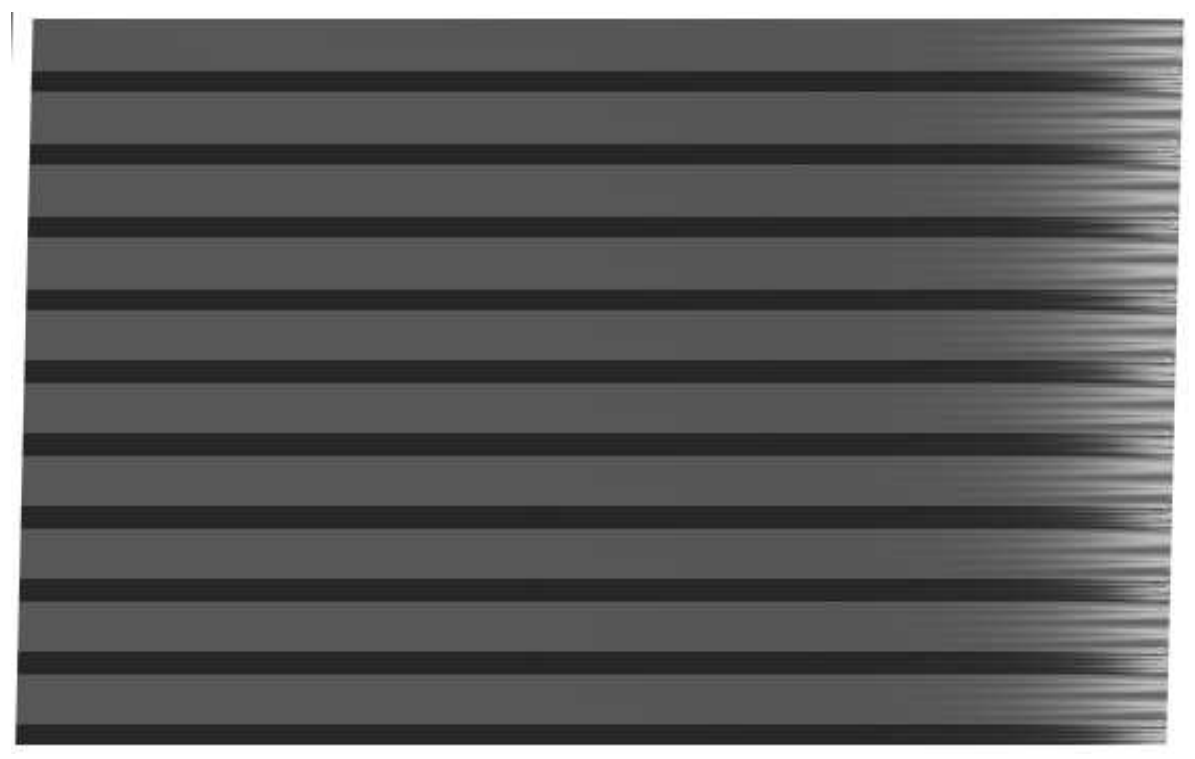

Fig. 4. Branched needles. Parameters: $\lambda=.3, L_{1}=.0673, L_{2}=1, N_{\text {layers }}=10, \quad N_{1}=200$, $N_{2}=400, d_{\lambda}=(.00114, .00107)$, and niter $=400$.

of elements of width $h_{1}$ near the laminate-single variant interface $\Gamma_{r}$ on which the deformation is linearly interpolated. To better visualize the experimental result of the needle-like microstructure, we put a band of the homogeneous deformation on the right side of the laminate-single variant interface. We observe in Fig. 3 a bent needle-like microstructure that is in qualitative agreement with the experimental result of Chu and James in Fig. 1. Our computational evidence found that the bending structure was dependent on the constant vector $d_{\lambda}$ in (3), as well as on the aspect ratio of the underlying domain and on the number of layers in the boundary data on $\Gamma_{l}$.

The second computational result is shown in Fig. 4. In this computation, the constant vector $d_{\lambda}$ in (3) was determined by assuming that the extension of the average laminated deformation $\left[\lambda Q U_{1}+(1-\lambda) U_{2}\right] x$ from the boundary data on $\Gamma_{l}$ into $\Omega_{\lambda}$ is continuous with the boundary data on $\Gamma_{r}$, so

$$
\left[\lambda Q U_{1}+(1-\lambda) U_{2}\right] x=R_{\lambda} U_{2} x+d_{\lambda} \quad \text { for all } x \in \Gamma_{r}
$$


Hence, we obtain from the compatibility condition (2) that

$$
d_{\lambda}=-L_{1}\left(m_{\lambda} \cdot n^{\perp}\right) b_{\lambda} \quad \text { where } \quad n^{\perp}=\frac{\sqrt{2}}{2}\left(\begin{array}{c}
1 \\
-1
\end{array}\right)
$$

We note that the choice of $d_{\lambda}$ in Fig. 3 is given by the formula (4) with $\lambda=.5$ and $L=.065$. The initial deformation in the conjugate gradient iteration in Fig. 4 was the same as used for the previous computation Fig. 3 .

In Fig. 4, we see that the needles split and get finer and finer near the laminate-single variant interface $\Gamma_{r}$. This can be explained by the necessity that an energy-minimizing deformation be compatible with a laminate of scale $L_{2} / N_{\text {layers }}$ on the left boundary $\Gamma_{l}$ and be compatible with a laminate having an infinitesimal scale at the right boundary $\Gamma_{r}$. By the compatibility condition (2), finer and finer needles can better approximate the homogeneous state, hence lower the energy of the transition layer near the laminate-single variant interface $\Gamma_{r}$. However, the fixed number of layers $N_{\text {layers }}$ in the boundary data on $\Gamma_{l}$ determines a macroscopic length scale $L_{2} / N_{\text {layers }}$ that penalizes fine scale twins away from the laminatesingle variant interface $\Gamma_{l}$. As a result, the layers branch to satisfy the mutually exclusive energy-minimizing requirements for the two boundaries $\Gamma_{r}$ and $\Gamma_{l}$. We note that the fineness of our computed branched needles is limited by the finite element mesh size.

Using a strain-gradient model, Kohn and Müller have shown that twin branching is a result of the balance of the elastic energy and the surface energy across twin boundaries [16]. In contrast, our computation indicates that twins can also branch when there is a fixed length scale in the boundary data.

The last computational result is shown in Fig. 5. The constant vector $d_{\lambda}$ was determined in the same way as in the previous computation Fig. 4. However, the initial deformation is identical to the homogeneous deformation in the boundary data over the entire computational domain except in a thin strip near the boundary $\Gamma_{l}$ where the homogeneous deformation is interpolated to the boundary data. 


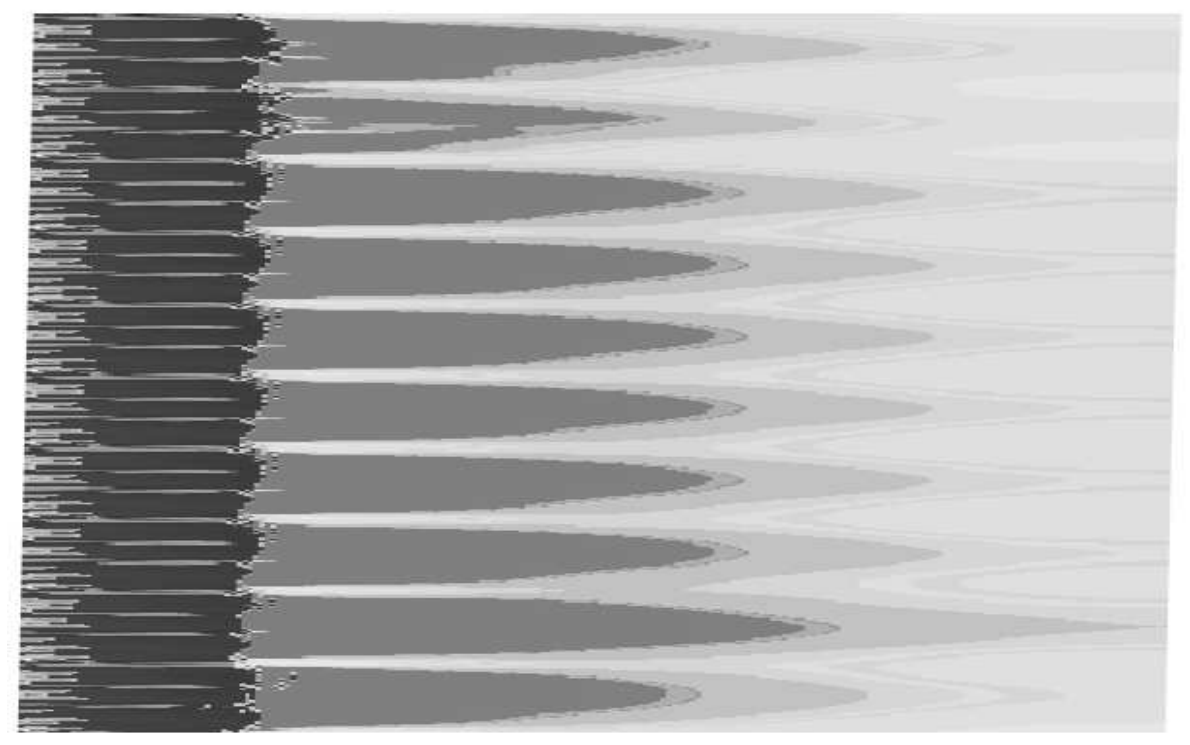

Fig. 5. Multiple arrays of needles. Parameters: $\lambda=.3, L_{1}=.068, L_{2}=1, N_{\text {layers }}=10$, $N_{1}=200, N_{2}=400, d_{\lambda}=(.00115, .00108)$, and niter $=800$.

We see from Fig. 5 that a secondary array of needles is formed and that a change of microstructural scales occurs. This computational result may have some relation to the complex microstructure that has been experimentally observed by several researchers $[17,18]$.

\section{Conclusion.}

We have developed a geometrically nonlinear continuum model for the needle-like microstructure near the interface between twinned layers and a pure variant of martensite. Our computations show that this model can be used to simulate the branching and bending of twinned layers at an interface.

\section{Acknowledgments.}

We thank R. James and R. Kohn for many helpful discussions, and C. Chu and R. James for providing Fig. 1. This work was supported in part by NSF DMS 95-05077, by AFOSR F49620-98-1-0433 and AF/F 49620-96-1-0212, by ARO DAAG55-98-1-0335, by ARPA/URI/ONR N00014-92-J-1890, by the Institute for Mathematics and Its Applications, and by the Minnesota Supercomputer Institute. 


\section{REFERENCES}

[1] C. Chu and R. James. J. de Physique IV, 5:143-149, 1995. ICOMAT-95.

[2] J Ball, C. Chu, and R. James. J. de Physique IV, 5:245-251, 1995. ICOMAT-95.

[3] R. Abeyarantne, C. Chu, and R. James. Phil. Mag. A, 73:457-497, 1996.

[4] C. Chu. PhD thesis, University of Minnesota, 1993.

[5] J. Ball and R. James. Arch. Rat. Mech. Anal., 100:13-52, 1987.

[6] M. Chipot and D. Kinderlehrer. Arch. Rat. Mech. Anal., 103:237-277, 1988.

[7] J. Ball and R. James. Phil. Trans. R. Soc. Lond. A, 338:389-450, 1992.

[8] J. Ericksen. J. Solids and Structures, 22:951-964, 1986.

[9] M. Luskin. Acta Numerica, 5:191-257, 1996.

[10] K. Bhattacharya, B. Li, and M. Luskin. Technical Report 1566, IMA, 1998.

[11] R. James, 1993. personal communication.

[12] R. James, R. Kohn, and T. Shield. J. de Physique IV, 5:253-259, 1995. ICOMAT-95.

[13] M. Killough. PhD thesis, NYU, 1998.

[14] B. Li. PhD thesis, University of Minnesota, 1996.

[15] Philippe Ciarlet. The Finite Element Method for Elliptic Problems. North-Holland, Amsterdam, 1978.

[16] R. Kohn and S. Müller. Philosophical Magazine, 66A:697-715, 1992.

[17] R. B. Flippen and C. W. Haas. Solid State Communications, 13:1207-1209, 1973.

[18] S. W. Meeks and B. A. Auld. Appl. Phys. Lett., 47:102-104, 1985. 